\title{
PRÁTICA DE FUTEBOL E FATORES SOCIODEMOGRÁFICOS ASSOCIADOS EM ADOLESCENTES
}

\author{
DR. DIEGO AUGUSTO SANTOS SILVA
}

Professor Adjunto do Centro de Desportos (CDS) da Universidade Federal de Santa Catarina (UFSC)

(Florianópolis - Santa Catarina - Brasil), Doutorado em Educação Física pela Universidade

Federal de Santa Catarina (UFSC) (Florianópolis - Santa Catarina - Brasil).

E-mail: diegoaugustoss@yahoo.com.br

\section{DR. ROBERTO JERÔNIMO DOS SANTOS SILVA}

Professor Adjunto do Departamento de Educação Física da Universidade Federal de Sergipe (UFS)

(São Cristovão - Sergipe - Brasil), Doutorado em Ciências da Saúde pela Universidade

Federal de Sergipe (São Cristovão - Sergipe - Brasil),

E-mail: rjeronimoss@gmail.com

\section{DR. EDIO LUIZ PETROSKI}

Professor titular do Centro de Desportos (CDS) da Universidade Federal de Santa Catarina (UFSC)

(Florianópolis - Santa Catarina - Brasil), Doutorado em Educação Física pela Universidade

Federal de Santa Maria (UFSM) (Santa Maria - Rio Grande do Sul - Brasil).

E-mail: petroski@cds.ufsc.br

\section{RESUMO}

Objetivou-se verificar a associação entre a prática de futebol com fatores sociodemográficos em escolares. A amostra foi composta por 906 estudantes de escolas públicas de Aracaju, Sergipe, Brasil, com média de 15, II (DP=2,19) anos de idade. Utilizou-se a regressão de Poisson para o cálculo da razão de prevalência (RP) e intervalos de confiança de $95 \%$ (IC95\%). Dos estudantes investigados, 49, I\% não praticavam futebol. Os escolares com maiores probabilidades de não praticar futebol foram os do sexo feminino (RP: 3,28; IC95\%: 2,59-4, 15) e do ensino médio (RP: 1,45; IC95\%: 1, 10-1,92). Quase metade dos estudantes não pratica futebol e, independente da faixa etária e nível econômico, em estudantes do sexo feminino e do ensino médio a probabilidade de prática na modalidade é ainda menor.

PALAVRAS-CHAVE: Futebol; atividade motora; estudantes; educação física e treinamento. 
Há várias definições de esporte, os autores entendem que esporte é uma atividade social e como tal, intrinsecamente política (OURIQUES, 20 I 0). Além disso, concordam que o esporte é visto como um antídoto para a ocupação do tempo livre, numa concepção que indica uma suposta linearidade entre a falta de lazer e o mundo do crime (MELO, 2005). Porém, devido à natureza do presente estudo, os autores se apoiaram na seguinte definição de Barbanti (2006, p. 57):

Esporte é uma atividade competitiva institucionalizada que envolve esforço físico vigoroso ou o uso de habilidades motoras relativamente complexas, por indivíduos, cuja participação é motivada por uma combinação de fatores intrínsecos e extrínsecos.

A atividade física, por sua vez, é considerada qualquer movimento corporal produzido pela musculatura esquelética que resulte em gasto energético acima dos níveis de repouso (CASPERSEN; POWELL; CHRISTENSEN, 1985). Com estes conceitos pode-se afirmar que o esporte é uma atividade física, porém nem toda atividade física é um esporte.

Com mais de 256 milhões de praticantes, o futebol é considerado o esporte mais popular do mundo, e sua popularidade continua crescendo (FEDERATION INTERNATIONAL DE FOOTBALL ASSOCIATION, 2007). Nos Estados Unidos, por exemplo, de 1999 para 2003 o número de adolescentes que praticavam futebol passou de 305.000 para 658.000 (NATIONAL FEDERATION OF STATE HIGH SCHOOL ASSOCIATIONS, 2005). AAcademia Americana de Pediatria estima que o crescimento de jovens que praticam futebol, naquele país, é em torno de $20 \%$ ao ano (AMERICAN ACADEMY OF PEDIATRICS, 2000).

No Brasil, o futebol movimenta muito dinheiro e é uma modalidade esportiva com muitos adeptos, o que faz o Brasil ser reconhecido como "o país do futebol" (GRIJÓ, 200I). Não existe uma estimativa de quantos adolescentes praticam futebol no país, todavia, acredita-se que milhares de jovens jogam esta modalidade esportiva no tempo livre (PAIM, 200 I). Além disso, este esporte desperta interesse de ascensão social nos milhares de adolescentes que praticam esta modalidade nas comunidades carentes, colégios e clubes do país (ALCÂNTARA, 2006).

O futebol é considerado uma prática de atividade física de intensidade vigorosa (AINSWORTH et al., 2000), e o Centro de Controle e Prevenção de Doenças CDC (CENTERS FOR DISEASE CONTROL AND PREVENTION, 1997) sugeriu que sua prática fosse incentivada nas escolas e na comunidade como uma maneira de promover a atividade física, durante o lazer, entre crianças e adolescentes, pois esta modalidade poderia ser uma maneira prazerosa dos jovens aumentarem os níveis de prática e resultar em melhoras na aptidão física, relações sociais, produtividade e rendimento acadêmico. 
Além destes benefícios relatados pelo CDC, pesquisadores divulgaram que adolescentes praticantes de futebol no lazer sofrem menos dores nas costas (SKOFFER; FOLDSPANG, 2008) e apresentam um perfil lipídico mais favorável em comparação aos seus pares que não praticam (BERGERON, 2007). Uma revisão sistemática demonstrou que o esporte, se praticado pelos adolescentes dentro dos limites do organismo, traz benefícios aos mais variados órgãos e sistemas do corpo humano: cardiovascular, respiratório, muscular, esquelético, cartilaginoso e endócrino (ALVES; LIMA, 2008).

Embora estas evidências sustentem a importância da prática de atividades físicas como o futebol, estudos em diferentes cidades do Brasil reportaram que mais de $60 \%$ dos adolescentes não estão engajados em atividades físicas moderadas e vigorosas (DA SILVA et al., 2005; SILVA et al., 2009), e uma parcela significativa destes jovens não têm a intenção de se engajar (PELEGRINI; PETROSKI, 2009). Alguns fatores estão sendo extensivamente estudados como determinantes da prática de atividades físicas durante o lazer, como os ambientais (PANTER; JONES; VAN SLUIJS, 2008), comportamentais (DA SILVA et al., 2005) e sociodemográficos (SILVA et al., 2009), o que vem resultando em programas de intervenção cada vez mais eficientes (BARROS et al., 2008). Entretanto, tais estudos não investigaram os fatores associados à prática de futebol, que pode ser uma alternativa prazerosa e barata para aumentar os níveis de atividade física, além de ter grande aceitação e popularidade no Brasil.

Tendo em vista os benefícios que a prática do futebol pode trazer à saúde, a escassez de publicações que identifiquem os fatores associados à prática desta modalidade e que tal prática está relacionada ao lazer dos jovens estudantes, o presente estudo tem como objetivo descrever os fatores sociodemográficos associados à prática de futebol no lazer em escolares.

\section{MATERIAIS E MÉTODOS}

Este estudo de delineamento transversal é parte do projeto intitulado Crescimento, composição corporal e desempenho físico em crianças e adolescentes do município de Aracaju (SE) e foi aprovado pelo Comitê de Ética da Universidade Tiradentes, sob número 12/04.

\section{AMOSTRA}

Foram definidos como elegíveis para inclusão na pesquisa todos os estudantes matriculados na rede de ensino regular, na faixa etária de 10 a 19 anos. Para se 
obter amostragem representativa de indivíduos do ensino fundamental e médio da rede estadual de Aracaju, SE, realizaram-se os procedimentos em duas etapas. $\mathrm{Na}$ primeira, houve a divisão do município em quatro regiões (Centro, Norte, Sul e Oeste). Em cada região, selecionou-se de forma não-probabilística a escola com maior número de alunos matriculados, totalizando quatro escolas. Na segunda etapa, em cada uma das quatro escolas visitadas, sortearam-se as turmas necessárias para atingir o número representativo de escolares de cada região, respeitando-se a proporcionalidade nas séries do ensino fundamental e médio. Todos os indivíduos presentes em sala de aula, no dia da coleta, foram convidados a participar do estudo.

Para o cálculo do tamanho da amostra, adotaram-se as orientações sugeridas por Luiz e Magnanini (2000). Adotou-se um erro tolerável de 4\%, nível de confiança de $95 \%$ e o efeito do desenho (deff) de I,5. Considerou-se uma prevalência de inatividade física de 70\% (SILVA et al., 2009). Segundo a Secretaria de Estado da Educação do Sergipe, em 2006, estavam matriculados 34.21 I alunos no ensino fundamental e 21.73 I no ensino médio de Aracaju. Estimou-se uma amostra de 957 estudantes, sendo 480 do ensino fundamental e 477 do médio.

Foram considerados como perdas, os estudantes sem consentimento dos pais/responsáveis ( $<18$ anos de idade), os que não assinaram o Termo de Consentimento Livre e Esclarecido ( $\geq 18$ anos) e aqueles que se negaram a responder os questionários. A coleta de dados foi executada no ano de 2006, em duas visitas para cada escola. Os questionários foram aplicados coletivamente, por equipe formada pelo pesquisador principal e quatro assistentes.

\section{INSTRUMENTOS E PROCEDIMENTOS}

Para identificar o comportamento em relação à prática de futebol, utilizou-se o item um (I ) do questionário PAQ-C (CROCKER et al., 1997). Tal questionário já foi empregado em pesquisas no Brasil por ser considerado adequado e válido para jovens na faixa etária do presente estudo (DA SILVA et al., 2005). Os indicadores de validade deste questionário para crianças e adolescentes de diferentes características étnicas foram considerados satisfatórios (MOORE et al., 2007). O questionário foi traduzido e adaptado a fim de excluir atividades físicas não praticadas no Brasil (SILVA; MALINA, 2000). O item um ( I ) que foi analisado no presente estudo restringe-se a avaliar as atividades que o sujeito realizou nos últimos sete dias anteriores à resposta. Dentre as atividades, têm-se a prática de futebol, de modo que o respondente tem que indicar em quantos dias da semana realizou esta prática. As opções de resposta são: I) "Nenhuma vez na semana"; 2) "I-2 vezes na semana"; 3) "3-4 vezes na semana"; 4) "5-6 vezes na semana"; 5) "7 ou mais vezes na semana". Para as análises 
de associação, as opções de resposta foram tratadas de forma dicotômica, sendo que a primeira categoria foi "Nenhuma vez na semana", e a segunda categoria foi formada por todas as outras opções de resposta.

As variáveis sociodemográficas analisadas foram: sexo (masculino e feminino), faixa etária (10-12; 13-15 e 16-19 anos), nível de escolaridade que estava cursando no momento da pesquisa (fundamental e médio) e nível econômico. $\bigcirc$ nível econômico foi identificado pelo questionário da Associação Brasileira de Empresas de Pesquisa - ABEP (ASSOCIAÇÃO BRASILEIRA DE EMPRESAS DE PESQUISA, 2003). A ABEP utiliza um sistema de pontos que, somados, servem para dividir a população brasileira em classes econômicas conforme sua capacidade de compra. As classes dos critérios adotados pela ABEP (2003) são cinco: "A", "B", "C", "D" e "E" por ordem decrescente de poder de compra. No presente estudo, o nível econômico foi dividido em três classes: "Alta" ("A" + "B"); "Média" ("C"); "Baixa” ("D" + "E").

\section{TRATAMENTO ESTATÍSTICO}

Para caracterização da amostra quanto à prática de futebol e variáveis sociodemográficas, utilizaram-se a estatística descritiva (frequência absoluta e relativa) e o teste qui-quadrado para comparar as proporções. Utilizou-se a regressão de Poisson (bruta e ajustada) para examinar as associações entre o desfecho com aspectos sociodemográficos, estimando-se razões de prevalências e os intervalos de confiança. Todas as variáveis sociodemográficas foram introduzidas no modelo de regressão. O nível de significância foi estabelecido em 5\% ( $p<0,05$ ou IC95\%).

\section{RESULTADOS}

Ao considerar os critérios de exclusão, a amostra final foi formada por 906 estudantes da rede pública de Aracaju, sendo 454 do ensino fundamental e 452 do médio, com média de 15, I | (desvio padrão=2, 19) anos de idade.

Na Figura I, verifica-se a distribuição dos estudantes em relação à frequência semanal de prática de futebol. A maior parte não pratica futebol em nenhum dia na semana $(49,1 \%)$. 


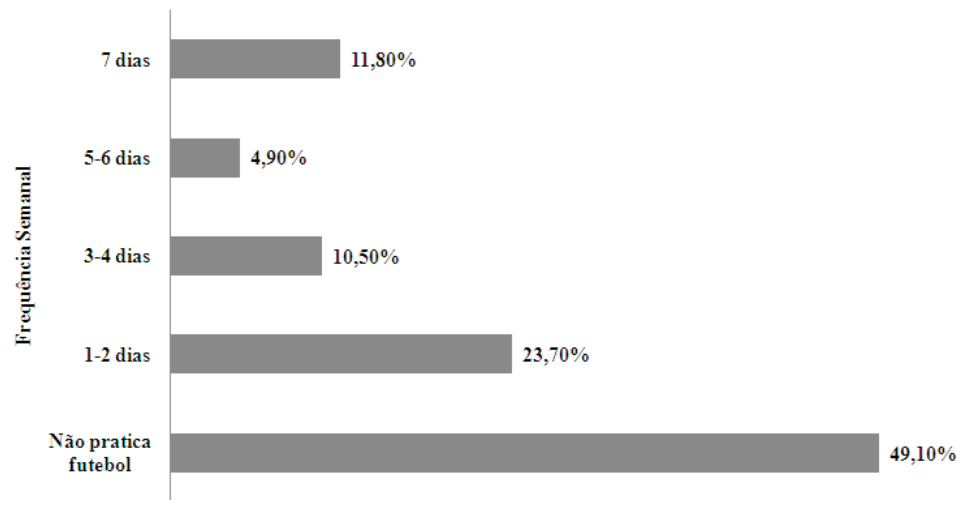

Figura I. Distribuição dos estudantes em relação à frequência semanal de prática de futebol. Aracaju, Sergipe, Brasil $(n=906)$.

Na Tabela I, observa-se que mais estudantes do sexo feminino $(7 \mathrm{I}, 4 \%)$ que do masculino (21,3\%) declararam não praticar futebol. O mesmo comportamento foi observado nos estudantes de 16 - 19 anos (59,0\%), em comparação às outras idades e nos alunos que estavam cursando o ensino médio (61,5\%), em comparação aos que cursavam o ensino fundamental $(36,8 \%)(p<0,05)$.

Tabela I. Distribuição da amostra em relação à prática de futebol e variáveis sociodemográficas.

Aracaju, Sergipe, Brasil $(n=906)$

\begin{tabular}{|c|c|c|c|c|}
\hline \multirow{2}{*}{ Variável } & \multicolumn{3}{|c|}{ Pratica de Futebol } & \multirow{2}{*}{ p-valor } \\
\hline & $\begin{array}{l}\text { Amostra } \\
\mathrm{n}\end{array}$ & $\begin{array}{l}\text { Sim } \\
\%(n)\end{array}$ & $\begin{array}{l}\text { Não } \\
\% \text { (n) }\end{array}$ & \\
\hline \multicolumn{5}{|l|}{ Sexo } \\
\hline Masculino & 403 & $78,7(3 \mid 7)$ & $21,3(86)$ & \multirow[t]{2}{*}{$<0,00$ । } \\
\hline Feminino & 503 & $28,6(144)$ & 71,4 (359) & \\
\hline \multicolumn{5}{|l|}{ Faixa etária } \\
\hline $10-12$ & 145 & $70,3(102)$ & $29,7(43)$ & \multirow[t]{3}{*}{$<0,00$ I } \\
\hline $13-15$ & 334 & $55, I(184)$ & $44,9(150)$ & \\
\hline $16-19$ & 427 & $41,0(175)$ & $59,0(252)$ & \\
\hline \multicolumn{5}{|l|}{ Nível econômico } \\
\hline Alto & 168 & $54,8(92)$ & $45,2(76)$ & \multirow[t]{3}{*}{0,536} \\
\hline Médio & 551 & $50, I(276)$ & $49,9(275)$ & \\
\hline Baixo & 187 & 49,7 (93) & $50,3(94)$ & \\
\hline \multicolumn{5}{|l|}{ Escolaridade } \\
\hline Fundamental & 454 & $63,2(287)$ & $36,8(167)$ & \multirow[t]{2}{*}{$<0,00$ । } \\
\hline Médio & 452 & $38,5(174)$ & $61,5(278)$ & \\
\hline
\end{tabular}


A associação entre a prática de futebol com as variáveis sociodemográficas pode ser observada na Tabela 2. Na análise bruta, a variáveis que se associaram com o fato dos adolescentes não praticarem futebol foram: sexo, faixa etária e o nível de escolaridade. Na análise ajustada para todas as variáveis, os resultados indicaram que estudantes do sexo feminino (RP: 3,28; IC95\%: 2,59-4, I 5) e os que cursavam o ensino médio (RP: I,45; IC95\%: I , I0- I,92) foram os que apresentaram maior probabilidade de não praticar futebol.

Tabela 2. Razão de prevalência bruta e ajustada entre o fato de não praticar futebol no lazer com variáveis sociodemográficas. Aracaju, Sergipe, Brasil $(n=906)$

\begin{tabular}{|c|c|c|c|c|c|c|}
\hline \multirow[t]{2}{*}{ Variável } & \multicolumn{3}{|c|}{ Bruto } & \multicolumn{3}{|c|}{ Ajustado } \\
\hline & $\mathrm{RP}$ & (IC 95\%) & p-valor & $\mathrm{RP}$ & (IC 95\%) & p-valor \\
\hline \multicolumn{7}{|l|}{ Sexo } \\
\hline Masculino & 1 & & $<0,00$ । & I & & $<0,00$ । \\
\hline Feminino & 3,35 & $(2,64-4,23)$ & & 3,28 & $(2,59-4,15)$ & \\
\hline \multicolumn{7}{|l|}{ Faixa etária } \\
\hline $10-12$ & 1 & & & 1 & & \\
\hline $13-15$ & $\mid, 51$ & $(1,08-2,13)$ & 0,016 & 1,38 & $(0,97-1,97)$ & 0,108 \\
\hline $16-19$ & 1,99 & $(1,44-2,75)$ & $<0,00$ । & $1,4 \mid$ & $(0,93-2,15)$ & 0,072 \\
\hline \multicolumn{7}{|c|}{ Nível econômico } \\
\hline Alto & । & & & । & & \\
\hline Médio & 1,10 & $(0,86-1,42)$ & 0,448 & $\mid, 01$ & $(0,78-1,30)$ & 0,930 \\
\hline Baixo & 1,11 & $(0,82-1,50)$ & 0,494 & 0,99 & $(0,74-1,35)$ & 0,986 \\
\hline \multicolumn{7}{|l|}{ Escolaridade } \\
\hline Fundamental & । & & $<0,00$ । & 1 & & 0,009 \\
\hline Médio & 1,67 & $(1,38-2,03)$ & & 1,45 & $(1,10-1,92)$ & \\
\hline
\end{tabular}

$R P$ - razão de prevalência; IC - intervalo de confiança.

\section{DISCUSSÃO}

presente estudo, conduzido em estudantes do ensino fundamental e médio da cidade de Aracaju, Sergipe, Brasil, teve como objetivo analisar a associação entre a prática de futebol com variáveis sociodemográficas. $\bigcirc$ principal achado deste estudo foi identificar os alunos com maiores probabilidades de não praticar futebol, o que pode ser útil no planejamento escolar, para que a direção da escola e professores de Educação Física programem iniciativas que estimulem a prática deste esporte durante o lazer. Assim, este estudo pode servir de parâmetro para futuras investigações em outras cidades do país. 
Os resultados evidenciados na cidade de Aracaju, SE, deixam claro que o futebol é um fenômeno esportivo entre os rapazes, e pode ser uma explicação pelos altos níveis de atividade física destes em comparação às moças (SILVA et al., 2009). Uma possível explicação para este achado pode ser histórica, pois, no Brasil, o futebol ainda é considerado como esporte violento e masculinizado, sendo a inserção dos meninos incentivada desde cedo pela família como forma de prática corporal (GOELLNER, 2005). Por outro lado, se o esporte analisado fosse dança ou ginástica, pode-se supor que as meninas seriam o grupo que mais praticaria tais modalidades. Logo, percebe-se que há questões culturais envolvidas na prática dos esportes.

Nos Estados Unidos encontrou-se resultado contrário, pois, em idade escolar, mais meninas praticam futebol que meninos (YARD et al., 2008), sendo, talvez, por isso, que este esporte é tão popular entre as mulheres daquele país. Ademais, estes achados indicam o nível de popularidade e competitividade deste esporte nos diferentes países, pois, no Brasil, o futebol masculino é considerado o mais vitorioso dentre todos os países, em contrapartida, nos Estados Unidos, este comportamento é verificado no futebol feminino.

Estudantes de 10 a 12 anos de idade foram os que mais praticavam futebol, no presente estudo, sendo que este número diminuiu com avançar da idade. Este resultado corrobora estudos que investigaram a prática de atividade física vigorosa e do esporte em adolescentes (NADER et al., 2008; SILVA et al., 2009). Embora diversos autores (ROWLAND, 2006; SEABRA et al., 2008) tenham procurado identificar as razões para esse declínio, permanece por esclarecer se é resultado de fatores biológicos ou ligados a envolvimento de natureza social. Em relação aos fatores biológicos, Rowland (2006) sugeriu que, no sistema nervoso central, existe um centro de controle com capacidade para governar a atividade física regular, sendo a hiperatividade das crianças e a natureza das suas brincadeiras capazes de despertar e manter a maior parte das atividades do cérebro. Em relação a fatores de âmbito social que poderão ajudar a compreender o declínio da atividade física, Seabra et al. (2008, p. 727) destacam que:

[... a alteração que se verifica ao longo da idade nos modelos que orientam os comportamentos dos adolescentes, pois, na infância, a família parece ser o primeiro e o mais poderoso agente socializador na transmissão de valores, comportamentos e normas, entretanto, a entrada na adolescência leva os jovens a desenvolverem um sentido de autonomia e de independência que os motiva a "separarem-se" dos pais. Essa "separação" traduz-se, geralmente, numa alteração dos seus modelos e valores, fato que conduz a uma transferência das influências da família para os pares, o que, por sua vez, pode influenciar os comportamentos e os estilos de vida saudáveis que o adolescente evidenciava até então [...]. 
presente estudo também encontrou que escolares do ensino médio apresentaram um risco maior de não praticar futebol que os do ensino fundamental. Resultados semelhantes foram observados por outros autores que investigaram a prática de atividade física vigorosa durante o lazer (LASHERAS et al., 200 I). Além do incremento da idade, que é observado entre os níveis de ensino, outra possível explicação para este achado pode ser o aumento da responsabilidade e dos afazeres, que vão se intensificando com o passar das séries de ensino. No estado de Sergipe, por exemplo, escolares do ensino médio realizam exames vestibulares anualmente, e este fato requer uma dedicação maior aos estudos, deixando em segundo plano a prática de atividades físicas.

Os achados do presente estudo podem contribuir para os gestores educacionais e para as escolas do município a direcionar a promoção do esporte, com campanhas e incentivos aos escolares com maiores riscos de não praticarem esta modalidade, como o sexo feminino e os do ensino médio. Além disso, sugere-se que este esporte seja oportunizado durante o lazer dos jovens, pois é uma modalidade de baixo custo, acessível a ambos os sexos e considerado uma prática prazerosa de atividade física vigorosa.

fato de a investigação ter ocorrido somente com as maiores escolas de cada região pode ser um empecilho para extrapolar os dados para todo o ensino fundamental e médio da cidade. Ademais, a coleta de dados foi realizada em 2006, de modo que já se passaram mais de cinco anos e, como a atividade física é um comportamento complexo, os resultados nos dias atuais pode não ser o mesmo. Porém, como até o presente momento nenhuma intervenção de promoção da atividade física e de esportes no estado de Sergipe foi desenvolvida, acredita-se que há uma forte tendência para que os resultados dos dias atuais sejam bem próximos. Além disso, o fato do questionário se reportar aos últimos sete dias pode ser considerado outra limitação, pois se houvesse algum estudante que, por alguma razão, tal como: lesão ortopédica, condições climáticas, por exemplo, não tenha praticado a modalidade na última semana, foi considerado integrante do grupo de risco. $\bigcirc$ delineamento transversal que impossibilita identificar se as associações encontradas com a prática de futebol são causais e não ter investigado a frequência com que os estudantes praticavam futebol durante os dias da semana, foram outras limitações deste estudo. Entretanto, a excelente taxa de resposta (94,6\%) que se obteve nas escolas contribuiu para a validade interna da pesquisa, diminuindo a chance da ocorrência de erros sistemáticos. 
Diante do exposto, pode-se concluir que metade dos estudantes da cidade de Aracaju não pratica futebol no lazer e, independente da faixa etária e nível econômico, os escolares com maiores probabilidades de não praticarem futebol foram os do sexo feminino e os do ensino médio.

Recomenda-se que o futebol seja oportunizado durante o lazer dos jovens estudantes, pois é uma modalidade de baixo custo, prazerosa e que pode aumentar os níveis de atividade física da população escolar.

\section{Soccer practice and associated sociodemographic factors in adolescents}

ABSTRACT: The objective of this study was to evaluate the association between soccer practice and sociodemographic factors in adolescents. The sample consisted of 906 public school students from Aracaju, Sergipe, Brazil, with a mean age of I 5.1 I $(S D=2.19)$ years. Poisson regression was used to calculate the prevalence ratio (PR) and $95 \%$ confidence interval (95\% Cl). Among the students investigated, $49.1 \%$ had not played soccer during the last 7 days. Adolescent girls (PR: 3.28; 95\% Cl: 2.59-4.15) and high school students (PR: 1.45; 95\% Cl: 1. 10-1.92) presented the highest probability of not playing soccer. Almost half the students do not play soccer and the probability is even lower among adolescent girls and high school students, irrespective of age group and socioeconomic status.

KEYWORDS: Soccer; motor activity; students; physical education and training.

\section{La práctica de fútbol y factores sociodemográficos asociados en adolescentes}

RESUMEN: Este estudio evaluó la asociación entre la práctica de fútbol con factores sociodemográficos en los escolares. La muestra está compuesta por 906 estudiantes de escuelas públicas en Aracaju, Sergipe, Brasil, con una media de 15, II (SD = 2,19) años de edad. Se utilizó la regresión de Poisson para el cálculo de la tasa de prevalencia (RP) y los intervalos de confianza del 95\% (IC95\%). De los estudiantes encuestados, el 49, I \% no practica el fútbol. Los niños con más probabilidades de no jugar al fútbol eran mujeres (RP: 3,28, IC95\%: 2,59 a 4, 15) y de la escuela secundaria (RP: I,45, IC95\%: 1, 10 - 1,92). Casi la mitad de los estudiantes no practican el fútbol y sin importar la edad y el nivel económico, las alumnas y los alumnos de la escuela secundaria probablemente a la práctica en el deporte es aún menor. PALABRAS CLAVE: Fútbol; actividad motora; estudiantes; educación y entrenamiento fisico.

\section{REFERÊNCIAS}

AINSWORTH, B. E. et al. Compendium of physical activities: an update of activity codes and MET intensities. Medicine and Science in Sports and Exercise, Hagerstown, v. 32, n. 9, p. S498-S504, sept. 2000. 
ALCÂNTARA, H. A magia do futebol. Estudos avançados, São Paulo, v. 20, n. 57, p. $297-$ 3।3, maio 2006.

ALVES, C.; LIMA, R. V. B. Impacto da atividade física e esportes sobre o crescimento e puberdade de crianças e adolescentes. Revista Paulista de Pediatria, São Paulo, v. 26, n. 4, p. 383-391, dez. 2008.

AMERICAN ACADEMY OF PEDIATRICS, COMMITTEE ON SPORTS MEDICINE AND FITNESS. Injuries in youth soccer: a subject review. Pediatrics, Springfield, v. I05, n. I, p. 659-66I, mar. 2000.

ASSOCIAÇÃO BRASILEIRA DE EMPRESAS DE PESQUISA. Critério de Classificação Econômica Brasil. 2003. Disponível em: <http://www.abep.org/codigosguias/ABEP_CCEB.pdf>. Acesso em: 10 dez. 2004.

BARBANTI, V. J. O que é esporte? Revista Brasileira de Atividade Física e Saúde, Pelotas, v. II, n. I, p. 54-58, jan. 2006.

BARROS, M.V.G. et al. Effectiveness of a school based intervention on physical activity for high school students in brazil: The Saude na Boa Project. Journal of Physical Activity \& Health, Champaign, v. 6, n. 2, p. 163-169, mar. 2009.

BERGERON, M. F. Improving health through youth sports: is participation enough? New Directions for Youth Development, San Francisco, v. 6, n. I I 5, p. 27-4I, oct. 2007.

CASPERSEN, C. J.; POWELL, K. E.; CHRISTENSON, G. M. Physical activity, exercise, and physical fitness: definitions and distinctions for health-related research. Public Health Reports, Washington, v. 100, n. 2, p. 126-131, mar. 1985.

CENTERS FOR DISEASE CONTROL AND PREVENTION. Guidelines for school and community programs to promote lifelong physical activity among young people. MMWR. Recommendations and reports: Morbidity and mortality weekly report. Recommendations and reports/Centers for Disease Control, Atlanta, v. 46, n. 6, p. I-36, mar 1997.

CROCKER, P. R. et al. Measuring general levels of physical activity: Preliminary evidence for the Physical Activity Questionnaire for Older Children. Medicine and Science in Sports and Exercise, Hagerstown, v. 29, n. 10, p. 1344-1349, oct. 1997.

DA SILVA, M. et al. Prevalência de fatores de risco cardiovascular em crianças e adolescentes da rede de ensino da cidade de Maceió. Arquivos Brasileiro de Cardiologia, São Paulo, v. 84, n. 5, p. 387-392, maio 2005.

FEDERATION INTERNATIONAL DE FOOTBALL ASSOCIATION. Football Worldwide 2000: official FIFA survey. 2007. Disponível em: < http://www.fifa.com/mm/document/fifafacts/ bcoffsurv/bigcount.statspackage_7024.pdf>. Acesso em: 12 jun. 2009.

GOELLNER, S. V. Mulheres e futebol no Brasil: entre sombras e visibilidades. Revista Brasileira de Educação Física e Esporte, São Paulo, v. 19, n. 2, p. |43- I 5 I, jun. 2005. 
GRIJÓ, F. COB divulga raio-X do esporte. Jornal do Brasil. 200I. Disponível em: <http:// jbonline.terra.com.br/jb/papel/esportes/200 I/05/2 I/joresp200 l 052 l 0 l 6.html >. Acesso em: 10 abr. 2010.

LASHERAS, L. et al. Factors associated with physical activity among Spanish youth through the National Health Survey. Preventive Medicine, New York, v. 32, n. 6, p. 455-464, jun. 200 I.

LUIZ, R. R.; MAGNANINI, M. M. F. A lógica da determinação do tamanho da amostra em investigações epidemiológicas. Cadernos Saúde Coletiva, Rio de Janeiro, v. 8, n. 2, p. 9-28, jul. 2000.

MELO, M. P. Esporte e juventude pobre: políticas públicas de lazer na Vila Olímpica Maré. Campinas: Autores Associados, 2005.

MOORE, J. B. et al. Validation of the physical activity questionnaire for older children in children of different races. Pediatric Exercise Science, Champaign, v. 19, n. I, p. 6-19, feb. 2007.

NADER, P. R. et al. Moderate-to-vigorous physical activity from ages 9 to 15 years. JAMA: The Journal of the American Medical Association, Chicago, v. 300, n. 3, p. 295-305, jul. 2008.

NATIONAL FEDERATION OF STATE HIGH SCHOOL ASSOCIATIONS. NFHS participation figures. 2005. Disponível em: <http://www.nfhs.org/scriptcontent/va_custom/participation_figures/>. Acesso em: 12 jun. 2009.

OURIQUES, N. Esporte e política. In: MATIELLO JÚNIOR, E.; CAPELA, P.; BREILHJ. (Org.). Ensaios alternativos Latino-Americanos de Educação Física, Esportes e Saúde. Florianópolis: Copiart, 2010. p. 4-7.

PELEGRINI, A.; PETROSKI, E. L. Inatividade física e sua associação com estado nutricional, insatisfação com a imagem corporal e comportamentos sedentários em adolescentes de escolas públicas. Revista Paulista de Pediatria, São Paulo, v. 27, n. 4, p. 366-373, dez. 2009.

PAIM, M. C. C. Fatores motivacionais e desempenho no futebol. Revista da Educação Físical UEM, Maringá, v. 12, n. 2, p. 73-79, jul. 2001.

PANTER, J. R.; JONES, A. P.; VAN SLUIJS, E. M. Environmental determinants of active travel in youth: A review and framework for future research. The International Journal of Behavioral Nutrition and Physical Activity, London, v. 5, n. I, p. 34, jun. 2008.

ROWLAND, T. Physical activity, fitness, and children. In: BOUCHARD, C.; BLAIR, S. N. H.; HASKELL, W. L. Physical activity and health. Champaign: Human Kinetics, 2006. p. 259-270.

SEABRA, A. F. et al. Determinantes biológicos e sócio-culturais associados à prática de atividade física de adolescentes. Cadernos de Saúde Publica, Rio de Janeiro, v. 24, n. 4, p. 721 -736, abr. 2008. 
SILVA, D. A. S. et al. Nível de atividade física e comportamento sedentário em escolares. Revista Brasileira de Cineantropometria e Desempenho Humano, Florianópolis, v. II, n. 3, p. 299-306, jul. 2009.

SILVA, R. C. R.; MALINA, R. M. Nível de atividade física em adolescentes do Município de Niterói, Rio de Janeiro, Brasil. Cadernos de Saúde Pública, Rio de Janeiro, v. I6, n. 4, p. 1091 - 1097, out. 2000.

SKOFFER, B.; FOLDSPANG, A. Physical activity and low-back pain in schoolchildren. European Spine Journal, Heidelberg, v. 7, n. 3, p. 373-379, mar. 2008.

WORLD HEALTH ORGANIZATION (WHO). The Second Decade: Improving Adolescent Health and Development. Geneve, 200 I. Disponível em: <http://www.who.int/child_adolescent_health/documents/frh_adh_98_I8/en/index.html> Acesso em: 04 mar. 2010.

YARD, E. E. et al. The epidemiology of United States high school soccer injuries, 2005-2007. The American Journal of Sports Medicine, Baltimore, v. 36, n. 10, p. 1930-1937, oct. 2008.

Recebido em: 16 fev. 2012 Aprovado em: 29 set. 2012

Endereço para correspondência:

Diego Augusto Santos Silva

Campus Universitário Reitor João David Ferreira Lima Centro de Desportos, Departamento de Educação Física Bairro Trindade Florianópolis - SC CEP: 88040-900 